\title{
Characterization of Salmonella isolates from municipal sewage, patients, foods, and animals in Greece using antimicrobial susceptibility testing and pulsed field gel electrophoresis
}

\author{
Theofilos Papadopoulos ${ }^{1,2}$, Antonios Zdragas², Georgia Mandilara ${ }^{1}$, Georgios Vafeas², Virginia Giantzi², \\ Evanthia Petridou ${ }^{3}$ and Alkiviadis Vatopoulos ${ }^{1}$ \\ 1. National Reference Centre for Salmonella, National School of Public Health \& Central Public Health Laboratory, Hellenic \\ Centre of Disease Control and Prevention, 16672 Vari, Greece; 2. Laboratory of Bacterial Diseases, Veterinary Research \\ Institute, National Agricultural Foundation, NAGREF Campus, 57001 Thermi, Greece; 3. Laboratory of Microbiology, \\ School of Veterinary Medicine, Aristotle University, University Campus, 54124 Thessaloniki, Greece. \\ Corresponding author: Theofilos Papadopoulos, e-mail: theofilos23@vet.auth.gr \\ AZ: zdragas@vri.gr, GM: gmandilara@esdy.edu.gr, GV: vafeas@vri.gr, VG: giantzi@yahoo.gr, \\ EP: epetridou@vet.auth.gr, AV: avatopoulos@esdy.edu.gr \\ Received: 26-12-2015, Accepted: 18-02-2016, Published online: 24-03-2016
}

How to cite this article: Papadopoulos T, Zdragas A, Mandilara G, Vafeas G, Giantzi V, Petridou E, Vatopoulos A. Characterization of Salmonella isolates from municipal sewage, patients, foods, and animals in Greece using antimicrobial susceptibility testing and pulsed field gel electrophoresis. Int J One Health 2016;2: 12-18.

\begin{abstract}
Aims: We aimed to compare Salmonella isolates from different sources using molecular and phenotypic methods, targeting better possibility of understanding the epidemiology of this organism in the Greek context with emphasis in municipal wastewater.

Materials and Methods: In this study, we used pulsed field gel electrophoresis (PFGE) in combination with antimicrobial susceptibility testing to analyze a total of 88 Salmonella Enterica isolates from municipal sewage $(\mathrm{n}=25)$, humans $(\mathrm{n}=36)$, animals $(n=24)$, and foods $(n=3)$ in Greece.

Results: The higher resistance rates were found to the following antimicrobials: streptomycin (59.1\%), tetracycline (47.7\%), nalidixic acid (46.6\%), ampicillin (37.5\%), and oxolinic acid (35.2\%). Resistance to ciprofloxacin was not observed; 22 isolates (25\%) were sensitive to all 9 antimicrobials, $36 \%, 25 \%$ and $12 \%$ of human, animal and wastewater origin, respectively, showing a significant difference. Salmonella ser. Hadar was the serovar with the highest resistance rates followed by Salmonella ser. Anatum and Salmonella ser. Typhimurium; Salmonella ser. Infantis strains were almost pansusceptible. Cluster analysis did not reveal close genetic relationship between human animal food and wastewater strains belonging to the same serovars. In most of the cases, distinct clusters were observed between human and non-human isolates indicating diversity and no epidemiological connection.
\end{abstract}

Conclusion: This study indicates that municipal wastewater would be of interest to further monitor the community's prevalence of subclinical or non-reported $S$. Enterica infections.

Keywords: Salmonella, wastewater, sewage, PFGE, antimicrobial resistance.

\section{Introduction}

Salmonella is the most important cause of foodborne outbreaks in the European Union (EU). In 2013, Salmonella was responsible for approximately $30 \%$ of all outbreaks reported to the European Food Safety Authority. It is a zoonotic pathogen with poultry, cattle, and pigs being the primary reservoirs [1]. Humans can acquire Salmonella infections through the consumption of contaminated foods as well as contaminated drinking water. A variety of food products have been described as vehicles for transmitting Salmonella infections to humans including beef, poultry, pork, eggs, cheese, and seafood as well as fruits

Copyright: Papadopoulos, et al. This article is an open access article distributed under the terms of the Creative Commons Attribution 4.0 International License (http://creativecommons. org/licenses/by/4.0/), which permits unrestricted use, distribution, and reproduction in any medium, provided you give appropriate credit to the original author(s) and the source, provide a link to the Creative Commons license, and indicate if changes were made. The Creative Commons Public Domain Dedication waiver (http:// creativecommons.org/ publicdomain/zero/1.0/) applies to the data made available in this article, unless otherwise stated. and vegetables [2]. Although wastewater and drinking water are treated to eliminate pathogenic microorganisms and prevent waterborne transmission, numerous studies indicate that conventional wastewater treatment does not guarantee their complete elimination [3]. Several studies investigating salmonellosis outbreaks have linked the use of water contaminated with human feces or animal manure to outbreaks [4-6].

Most nontyphoidal Salmonella infections (NTS) result gastro-enteritis and do not require treatment; severe invasive infections can also occur, which then requires antibiotic treatment. Drugs for NTS infections include fluoroquinolones, trimethoprim-sulfamethoxazole, ampicillin, or third generation cephalosporins [7]. The emergence and spread of resistant bacteria and the subsequent failure of treatment of infections is significant and increasing Public Health problem. Resistance to antimicrobials used to treat invasive Salmonella is also increasing, including extended spectrum cephalosporins and quinolones [8,9].

DNA fingerprinting techniques such as pulsedfield gel electrophoresis (PFGE), which is currently 
the gold standard for subtyping of salmonellae, are used in outbreak investigations to supplement phage typing data where further strain discrimination is required $[10,11]$. PFGE has been applied extensively in the epidemiological investigation and surveillance of Salmonella for the last two decades. Although PFGE is considered as time-consuming and labor-intensive [12], it is currently the most widely used molecular subtyping method for Salmonella and is routinely used in the United States and Europe.

We hypothesize that there is a detectable prevalence of Salmonella in human communities and that municipal wastewater reflects this prevalence. Furthermore, this prevalence may be influenced by the environment that surrounds communities such as animals and especially poultry.

The aim of this work was to study the epidemiology of this organism in the Greek context with emphasis in municipal wastewater. A total of 88 strains isolated from patients, animals, food products, and wastewater were characterized by (i) assessing phenotypic antimicrobial resistance profiles using disc diffusion method and (ii) by genotyping using PFGE after macrorestriction with $X b a I$, to evaluate the relationship of the strains.

\section{Materials and Methods}

\section{Ethical approval}

All samples were collected as per standard sample collection procedure without harming humans or animals.

\section{Sampling}

The Veterinary Research Institute of Thessaloniki (VRI) receives samples during monitoring programs and also veterinary samples. Between 1998 and 2000 a total of 25 strains from municipal sewage samples were isolated from VRI and were sent to National Veterinary Reference Laboratory in Chalkis (NRLVet) for serotyping. These samples were isolated from sewage in the area of municipality of Thessaloniki (Table-1). During 2008, National Reference Centre for

Table-1: Salmonella isolates included in this study.

\begin{tabular}{lcccc}
\hline \multirow{2}{*}{$\begin{array}{l}\text { Salmonella } \\
\text { serovars }\end{array}$} & \multicolumn{4}{c}{$\boldsymbol{N}$} \\
\cline { 2 - 5 } & Environment & Animal & Human & Total \\
\hline Abonus & 2 & 0 & 0 & 2 \\
Alamo & 1 & 0 & 0 & 1 \\
Anatum & 8 & 2 & 0 & 10 \\
Blockley & 3 & 4 & 8 & 15 \\
Braenderup & 1 & 0 & 0 & 1 \\
Bredeney & 1 & 3 & 0 & 4 \\
Corvallis & 1 & 0 & 0 & 1 \\
Hadar & $3 *$ & 9 & 9 & 21 \\
Infantis & 2 & 0 & 10 & 12 \\
Orion & 4 & 1 & 0 & 5 \\
Typhimurium & 1 & 5 & 9 & 15 \\
Zerifin & 1 & 0 & 0 & 1 \\
Total & 28 & 24 & 36 & 88 \\
\hline
\end{tabular}

*Food isolates from 2010
Salmonella received 597 clinical isolates from clinical microbiology laboratories all over Greece, 36 of these isolates were included in this study. These samples were randomly selected from patients that have been hospitalized for Salmonella in several Greek hospitals. Furthermore, a total of 24 isolates from poultry were isolated from the area of Thessaloniki in VRI between 1998 and 2000 and were sent to NRL-Vet for serotyping. Finally, three strains isolated from foods of animal origin (ready to eat chicken products) in 2010 were included in this study; these isolates were categorized as environmental (Table-1).

\section{Antimicrobial susceptibility testing}

Antimicrobial susceptibility testing was performed using the Kirby-Bauer disk-diffusion method, and the zones of inhibition obtained were interpreted according to the Clinical and Laboratory Standards Institute guidelines [13]. The strains were screened for resistance to 9 antibiotics. The antibiotic discs $(\mu \mathrm{g}$, Bio-Rad, Marnes-La-Coquette, France) used were ampicillin 10 (A), cefoperazone 30 (Cf), ciprofloxacin $5(\mathrm{Cp})$, gentamicin $10(\mathrm{G})$, nalidixic acid $30(\mathrm{Nx})$, oxolinic acid $10(\mathrm{Ox})$, streptomycin $10(\mathrm{~S})$, tetracycline $30(\mathrm{~T})$, and trimethoprim $5(\mathrm{Tm})$. Isolates were cultured in trypticase soy broth supplemented with $0.6 \%$ yeast extract and transferred to Mueller-Hinton agar (Difco Laboratories, Detroit, MI, USA). The results were interpreted following "The European Committee on Antimicrobial Susceptibility Testing" criteria, except from oxolinic acid as diameter of $19 \mathrm{~mm}$ or less was considered susceptible [14]. Escherichia coli ATCC 25922 and Salmonella ser. Enteritidis ATCC 13076 were used as control strains. Multidrug resistance was defined as previously proposed [15].

\section{PFGE}

Salmonella isolates belonging to serovars Anatum ( $S$. Anatum) $(\mathrm{n}=10)$, Blockley $(S$. Bockley) $(\mathrm{n}=15)$, Infantis ( $S$. Infantis) $(\mathrm{n}=12)$, Hadar $(S$. Hadar $)$ $(\mathrm{n}=21)$ and Typhimurium $(S$. Typhimurium) $(\mathrm{n}=15)$, were studied using PFGE as previously described with slight modifications [11]. Cells were grown overnight on blood agar plates at $37^{\circ} \mathrm{C}$. Each culture was suspended in TE buffer (100 mM Tris- $\mathrm{HCl}, 100 \mathrm{mM}$ ethylenediaminetetraacetic acid, $\mathrm{pH}$ 8.0). To prepare the agarose plugs, $10 \mu \mathrm{l}$ of proteinase $\mathrm{K}(2000 \mathrm{mg} / \mathrm{dL}$ stock) was added to $190 \mu$ of the adjusted cell suspension. Then, $200 \mu \mathrm{l}$ of melted 1\% Certified Megabase agarose (Biorad, Hamstead, UK) was added to the $200 \mu \mathrm{l}$ cell suspension/Proteinase $\mathrm{K}$ mixture and mixed gently. The mixture was immediately dispensed into wells of plug molds. The bacterial cells in the agarose plugs were lysed by treatment with a lysis solution containing $0.1 \mathrm{mg} / \mathrm{ml}$ Proteinase K (GIBCOBRL, Gaithersburg, MD), $50 \mathrm{mM}$ Tris- $\mathrm{HCl}$ ( $\mathrm{pH} 8.0$ ), $50 \mathrm{mM}$ EDTA, and $1 \%$ N-lauroylsarcosine, for $2 \mathrm{~h}$ at $54^{\circ} \mathrm{C}$. The plugs were digested with $40 \mathrm{U}$ of restriction enzyme XbaI (Promega Corp., Madison, WI) for $4 \mathrm{~h}$ at $37^{\circ} \mathrm{C}$. Digested fragments were resolved in a $1 \%$ 
SeaKem Gold agarose gel (Lonza, Rockland, Maine) in $0.5 \times$ Tris-Borate-EDTA (TBE) buffer using a contour-clamped homogeneous electric field (CHEF) apparatus (CHEF-DR III, Bio-Rad Laboratories, Richmond, CA). Electrophoresis was performed at $6 \mathrm{~V} / \mathrm{cm}$ with 2.2-63.8 s linear ramp time for $19 \mathrm{~h}$. Gels were cooled at $14^{\circ} \mathrm{C}$ throughout the run and then stained with ethidium bromide and destained with distilled water. Banding patterns were visualized by UV and photographed (Gel Doc 2000 Imager, Biorad). Salmonella ser. Braenderup H9812-PulseNet standard was used as a molecular weight marker after digestion with XbaI [16].

\section{Data and statistical analysis}

Statistical comparison between different antimicrobial susceptibility rates was analyzed by the chisquare test (Microsoft Excel, 2011). Fingerprinting profiles were examined using the BioNumerics 6.1 software with the Dice coefficient and clustering was based on the unweighted pair group average method (UPGA) with $1.5 \%$ optimization and $1.5 \%$ position tolerance. A cut off value of $80 \%$ similarity was assigned for clustering isolates.

\section{Results}

\section{Antimicrobial susceptibility testing}

The antibiotic resistance of Salmonella strains to nine antimicrobial agents is shown in Table-2. Overall, the highest resistance rates were found to the following antimicrobials: Streptomycin (59.1\%), tetracycline (47.7\%), nalidixic acid (46.6\%), ampicillin $(37.5 \%)$, and oxolinic acid $(35.2 \%)(\mathrm{p}<0.05)$. Resistance to ciprofloxacin was not observed; 22 isolates $(25 \%)$ were sensitive to all nine antimicrobials, $36 \%$ and $25 \%$, respectively, for human and animal isolates, but only $12 \%$ from wastewater isolates, showing a significant difference. Interestingly, 15 out of $36(41 \%)$ human and 13 out of $24(54 \%)$ animal isolates were multiresistant but only 6 out of 25 of wastewater isolates.

$S$. Hadar was the serovar with the highest resistance rates $(\mathrm{p}<0.05)$ with 14 out of $21(66.7 \%)$ strains being multiresistant. $S$. Blockley was also a serovar with high levels of multiresistance with 8 out of $15(53.3 \%)$ isolates. Serovar Infantis was almost susceptible to all the antimicrobials tested, only 2 strains out of $12(16.7 \%)$ showed resistance

Table-2: Antimicrobial resistance percentages of main Salmonella serovars.

\begin{tabular}{lcccccc}
\hline $\begin{array}{l}\text { Antimicrobial } \\
\text { agents }\end{array}$ & \multicolumn{5}{c}{ Number $\mathbf{( \% )}$ of resistant isolates } \\
\cline { 2 - 7 } & $\begin{array}{c}\text { Anatum } \\
(\mathbf{n = 1 0 )}\end{array}$ & $\begin{array}{c}\text { Blockley } \\
(\mathbf{n = 1 5 )}\end{array}$ & $\begin{array}{c}\text { Hadar } \\
\mathbf{( n = 2 1 )}\end{array}$ & $\begin{array}{c}\text { Infantis } \\
(\mathbf{n = 1 2 )}\end{array}$ & $\begin{array}{c}\text { Typhimurium } \\
(\mathbf{n = 1 5})\end{array}$ & $\begin{array}{c}\text { Total } \\
(\mathbf{n = 8 8}=\mathbf{1 5})\end{array}$ \\
\hline Ampicillin & $1(10)$ & $5(33.3)$ & $15(71.4)$ & $0(0)$ & $9(60)$ & $33(37.5)$ \\
Cefoperazone & $0(0)$ & $3(20)$ & $11(52.4)$ & $0(0)$ & $1(6.7)$ & $16(18.2)$ \\
Ciprofloxacin & $0(0)$ & $0(0)$ & $0(0)$ & $0(0)$ & $0(0)$ & $0(0)$ \\
Gentamycin & $1(10)$ & $0(0)$ & $0(0)$ & $0(0)$ & $4(26.7)$ & $5(5.7)$ \\
Nalidixic acid & $4(40)$ & $15(100)$ & $15(71.4)$ & $0(0)$ & $0(26,7)$ & $41(46.6)$ \\
Oxalinic acid & $4(40)$ & $6(40)$ & $14(66.7)$ & $0(0)$ & $5(33.3)$ & $31(35.2)$ \\
Streptomycin & $6(60)$ & $15(100)$ & $13(61.9)$ & $2(16.7)$ & $4(26.7)$ & $52(59.1)$ \\
Tetracycline & $4(40)$ & $13(86.7)$ & $13(61.9)$ & $0(0)$ & $9(60)$ & $42(47.7)$ \\
Trimethoprim & $0(0)$ & $0(0)$ & $0(0)$ & $0(0)$ & $2(13.3)$ & $4(4.5)$ \\
\hline
\end{tabular}

Table-3: Antimicrobial resistance profiles of Salmonella isolates included in this study.

\begin{tabular}{|c|c|c|c|c|c|c|c|c|c|c|c|c|c|}
\hline $\begin{array}{l}\text { Resistance } \\
\text { patterns }\end{array}$ & $\begin{array}{c}\text { Total } \\
(n=88)\end{array}$ & $\begin{array}{c}A b \\
(n=2)\end{array}$ & $\begin{array}{c}\text { Al } \\
(n=1)\end{array}$ & $\underset{(n=10)}{A n}$ & $\begin{array}{c}B I \\
(n=15)\end{array}$ & $\begin{array}{c}B r \\
(n=1)\end{array}$ & $\begin{array}{c}\text { Bre } \\
(n=4)\end{array}$ & $\begin{array}{c}\text { Cor } \\
(n=1)\end{array}$ & $\begin{array}{c}\text { Had } \\
(n=21)\end{array}$ & $\begin{array}{c}\text { Inf } \\
(n=12)\end{array}$ & $\begin{array}{c}\text { Or } \\
(n=5)\end{array}$ & $\underset{(n=15)}{\operatorname{Tm}}$ & $\begin{array}{c}\text { Zer } \\
(n=1)\end{array}$ \\
\hline$A$ & 1 & - & - & - & - & - & - & - & 1 & - & - & - & - \\
\hline $\mathrm{S}$ & 14 & 2 & - & 3 & - & 1 & - & 1 & - & 2 & 5 & - & - \\
\hline $\mathrm{T}$ & 1 & - & - & - & - & - & - & - & - & - & - & 1 & - \\
\hline $\mathrm{Tm}$ & 1 & - & - & - & - & - & - & - & - & - & - & 1 & - \\
\hline$A-N x$ & 3 & - & - & - & - & - & - & - & 3 & - & - & - & - \\
\hline A-Tm & 1 & - & - & - & - & - & - & - & - & - & - & 1 & - \\
\hline $\mathrm{S}-\mathrm{Nx}$ & 2 & - & - & - & 2 & - & - & - & - & - & - & - & - \\
\hline$A-S-T$ & 3 & - & - & - & - & - & - & - & - & - & - & 3 & - \\
\hline S-T-Nx & 5 & - & - & - & 5 & - & - & - & - & - & - & - & - \\
\hline $\mathrm{S}-\mathrm{T}-\mathrm{Ox}$ & 1 & - & 1 & - & - & - & - & - & - & - & - & - & - \\
\hline$A-N x-O x-C f$ & 2 & - & - & - & - & - & - & - & 1 & - & - & - & 1 \\
\hline A-S-T-Nx & 2 & - & - & - & 2 & - & - & - & - & - & - & - & - \\
\hline A-S-T-Tm & 2 & - & - & - & 2 & - & - & - & - & - & - & - & - \\
\hline S-T-Nx-Ox & 9 & - & - & 3 & 3 & - & - & - & 3 & - & - & - & - \\
\hline A-G-T-Nx-Ox & 5 & - & - & 1 & - & - & - & - & - & - & - & 4 & - \\
\hline A-S-T-Nx-Ox & 1 & - & - & - & - & - & - & - & - & - & - & 1 & - \\
\hline A-S-T-Nx-Ox-Cf & 13 & - & - & - & 3 & - & - & - & 10 & - & - & - & - \\
\hline
\end{tabular}

$\mathrm{T}=$ Tetracycline, Nx=Nalidixic acid, A=Ampicillin, $\mathrm{S}=$ Streptomycin, Tm=Trimethoprim, Cf=Cefoperazone, G=Gentamicin, $\mathrm{Ox}=$ Oxolinic acid, $\mathrm{Ab}=\mathrm{Abonus}, \mathrm{Al}=\mathrm{Alamo}, \mathrm{An}=$ Anatum, $\mathrm{Bl}=\mathrm{Blockley}, \mathrm{Br}=$ Braederup, Bre=Bredeney, Cor=Corvalis, Had=Hadar, Inf=Infantis, Or=Orion, Tm=Typhimurium, Zer=Zerifin 
to streptomycin. For $S$. Typhimurium and $S$. Anatum, 5 out of $14(35.7 \%)$ and 4 out of 10 isolates $(40 \%)$ respectively were multiresistant. 17 different patterns were observed in the 66 resistant isolates; of them, 43 showed resistance to more than two antibiotics (Table-3). The predominant resistance pattern was $\mathrm{S}$ and was found in 14 strains followed by A-S-T-Nx$\mathrm{Ox}-\mathrm{Cf}$ in $13, \mathrm{~S}-\mathrm{T}-\mathrm{Nx}-\mathrm{Ox}$ in nine strains and S-T-Nx and A-G-T-Nx-Ox resistance patterns in five strains, respectively.

\section{PFGE patterns}

\section{S. Anatum}

PFGE of XbaI digested chromosomal DNA from 10 isolates gave stable reproducible patterns consisting of 11-16 fragments. Seven patterns were identified. Two environmental isolates with R-type S-T$\mathrm{Nx}-\mathrm{Ox}$ shared the $\mathrm{XbAnO1}$ pulsotype, two animal isolates (susceptible to all antibiotics) shared $\mathrm{XbAnO7}$ pulsotype. Using a cut off value of $80 \%$ similarity five clusters were observed. The most common cluster (cluster D) consisted of 4 out of 10 isolates, two from animal and two from environmental origin (Figure-1).

\section{S. Blockley}

Macrorestriction with XbaI yielded 14 different patterns consisting of 11-17 fragments. Three major clusters (A, C, and D) were observed using a cut off value of $80 \%$ similarity. All human isolates belonged to clusters $\mathrm{C}$ and $\mathrm{D}$ and all the animal and environmental isolates belonged to different clusters.
Correlation between specific pulsotypes and R-types was not observed (Figure-2).

\section{S. Hadar}

PFGE patterns of XbaI-digested chromosomal DNA of $21 \mathrm{~S}$. Hadar isolates consisting of 10-15 fragments are shown on Figure-3. About 15 patterns were observed belonging to six clusters. Clusters $\mathrm{C}$ and $\mathrm{D}$ were predominant; $\mathrm{C}$ consisting of six out of nine human isolates sharing R-type (A-S-T-Nx-Ox-Cf) and $\mathrm{D}$ consisting of eight out of nine animal isolates, sharing different R-types. The three environmental strains isolated from foods in 2010 shared the same pulsotype (XbHd14) and R-type (A-Nx).

\section{S. Infantis}

Digestion of the $12 S$. Infantis strains with restriction enzyme XbaI demonstrated 11 patterns consisting of 10-15 fragments. 11 pulsed-field profiles were obtained, one main cluster (C) with five human isolates all susceptible to antimicrobials and seven individual types (Figure-4).

\section{S. Typhimurium}

About 10 PFGE types were demonstrated after digestion of $15 S$. Typhimurium strains consisting of 11-16 fragments. These types were classified into five clusters, cluster A consisted of five animal strains; four strains XbTyph01 (A-G-T-Nx-Ox R-type all isolated in 2000) and one XbTyph02 (susceptible, isolated in 1998). The human strains belonged to clusters

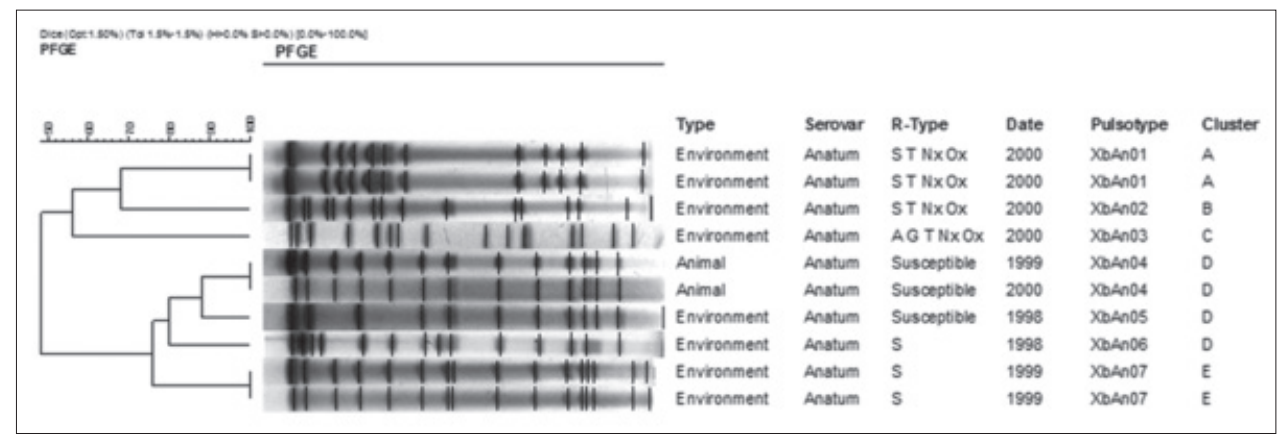

Figure-1: Dendrogram for Salmonella ser. Anatum.

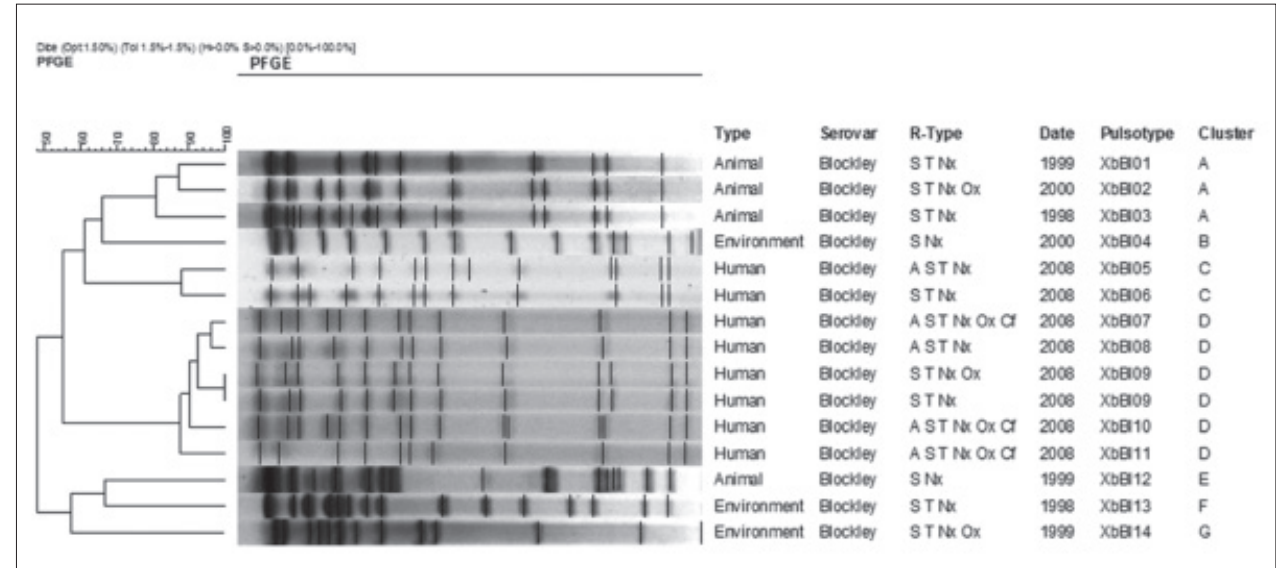

Figure-2: Dendrogram for Salmonella ser. Blockley. 


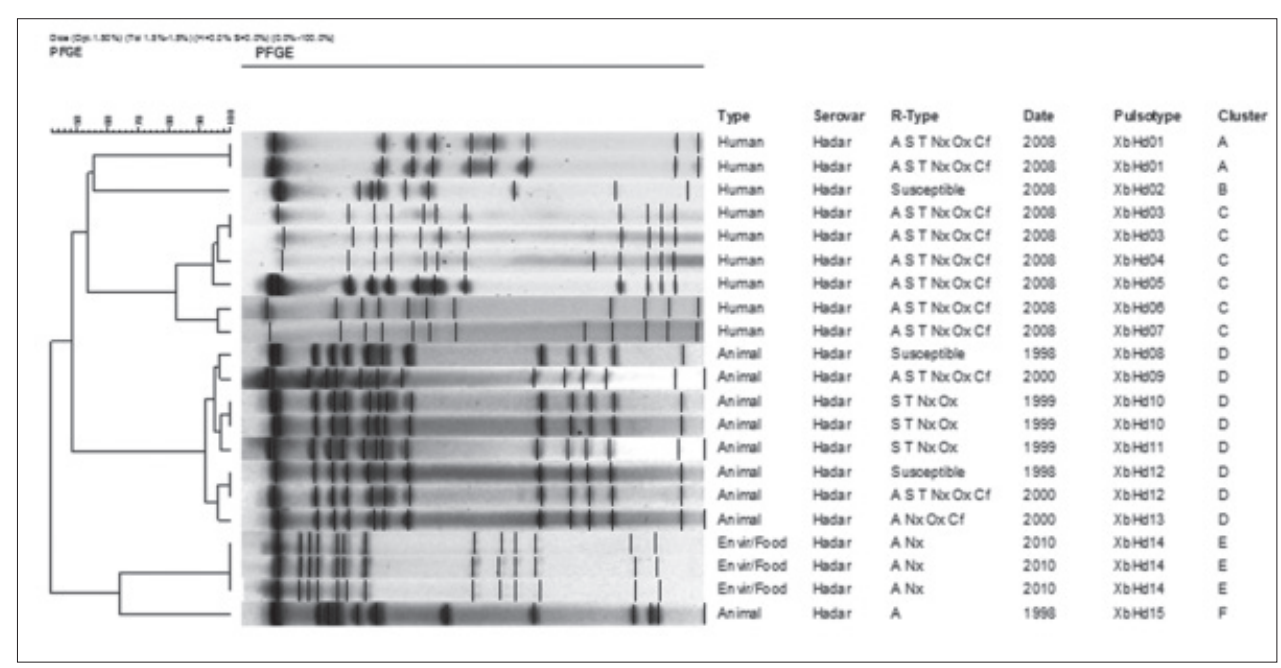

Figure-3: Dendrogram for Salmonella ser. Hadar.

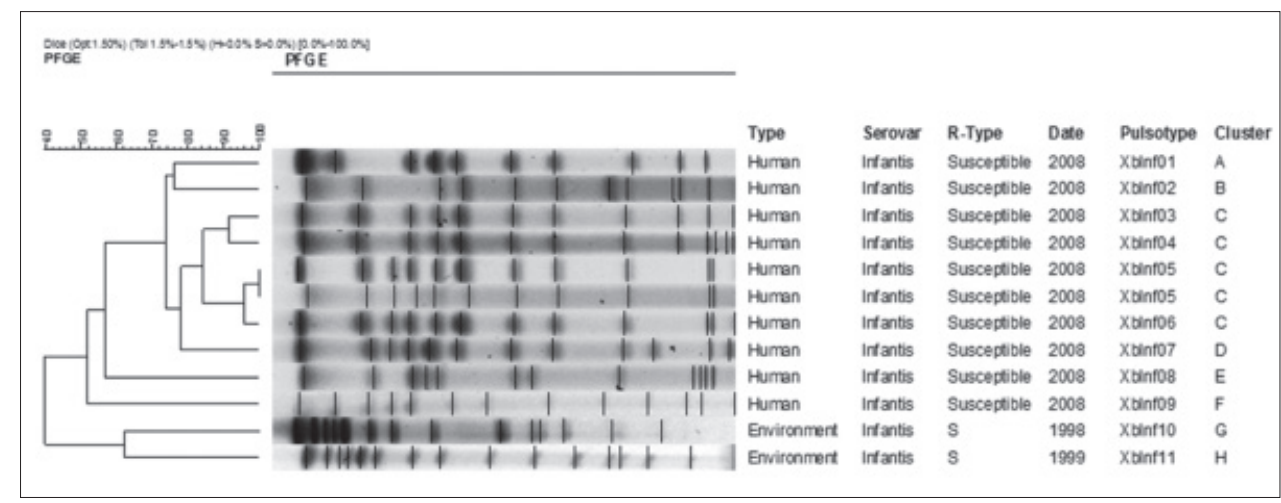

Figure-4: Dendrogram for Salmonella ser. Infantis.

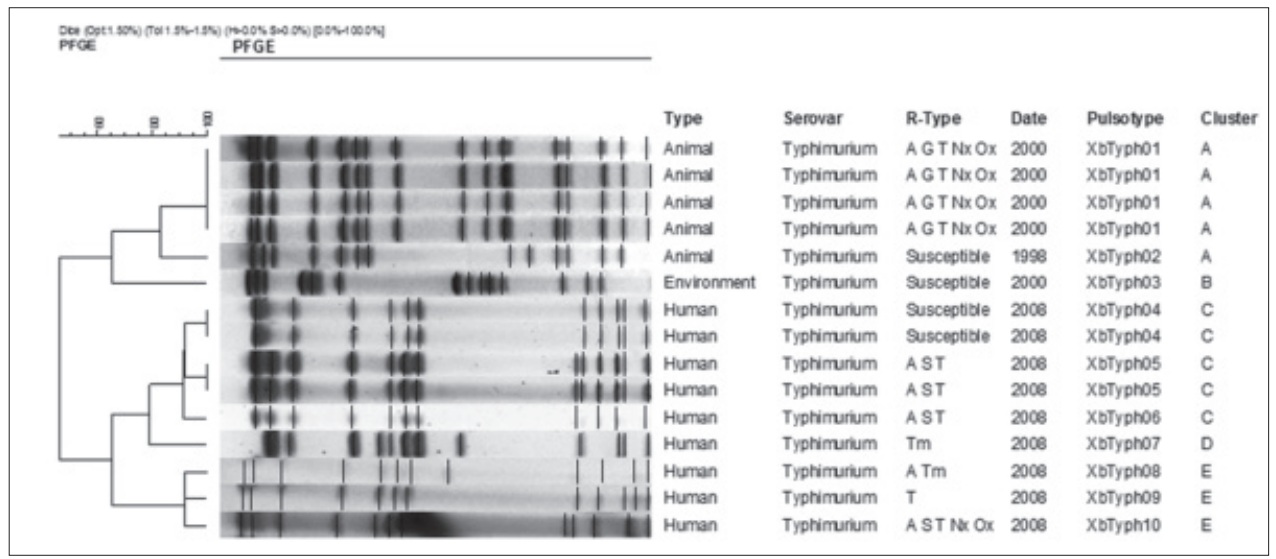

Figure-5: Dendrogram for Salmonella ser. Typhimurium.

C (five strains), D (one strain) and E (three strains) (Figure-5).

\section{Discussion}

To our knowledge, this is the first study that describes the population structure of Salmonella isolates based on PFGE types and drug resistance surveillance comparing human, animal and wastewater isolates in Greece. Even though the isolates do not cover the current period, we believe that the general condition has not changed substantially over the last years and the main conclusions are still an ongoing issue. However, this work underlines the need of establishing a monitoring system in wastewater facilities in Greece.

This study has shown that a wide variety of serovars and antimicrobial resistance patterns were observed in municipal wastewater. Several of the serotypes isolated in this study, including $S$. Typhimurium, $S$. Hadar, $S$. Infantis, $S$. Blockley and $S$. Anatum, have frequently been implicated in outbreaks or sporadic cases of human illness [17]. In Greece, as elsewhere in the world, $S$. Enteritidis and $S$. Typhimurium are the most common causes 
of human salmonellosis [18-21]. The main serovars found in both animal and human fecal samples from Greece included $S$. Hadar (from poultry), $S$. Typhimurium (from all the studied animal species) and $S$. Infantis. According to our study, the most common serovar detected in the wastewater samples was $S$. Anatum, which is a rare serovar and has been isolated in wastewater also in Spain [22]. Interestingly $S$. Enteritidis, the most common serovar in human and poultry in Greece and worldwide, has not been detected from wastewater samples in our study as in other studies [23]. In contrast, this serovar has been isolated in Portugal [24] and also in Spain from sea, river and fresh reservoirs [25].

The higher resistance levels were observed to ampicillin, streptomycin, tetracycline, and nalidixic acid, similar to those reported in EU in 2012, where varying levels of resistance were observed among NTS infections acquired in the different geographical regions [19]. Concerning human strains acquired from different countries within EU, the highest levels of resistance are being observed to ampicillin $(27.8 \%)$, streptomycin $(23.9 \%)$, sulfonamides $(29.2 \%)$, and tetracyclines $(30.2 \%)$. Resistance to ciprofloxacin was not observed; in line to our results, low rates of resistance to ciprofloxacin $(<3 \%)$ were observed from strains of human and animal origin in EU during 2007-2012. Interestingly resistance levels to ciprofloxacin are higher in isolates from other regions and particularly high in isolates originating in Asia $(22.7 \%)$, Africa $(20.5 \%)$ and Europe (non-EU/ EEA countries) $(19.5 \%$, ) [19]. Susceptibility to all antimicrobials demonstrated to $25 \%$ of the isolates, $36 \%$ of the human, $25 \%$ of the animal and $12 \%$ of the wastewater. However, resistance levels can also differ substantially between Salmonella serovars, and therefore, an in-depth analysis among every serovar is essential.

According to our study, multiresistant isolates were $39 \%(n=88)$, in line to previous studies in Greece concerning poultry and poultry products [26,27]. Multi-drug resistance is high among isolates from humans in EU (28.9\%) with the higher levels reported from Italy $(63.1 \%)$ and Hungary $(55.8 \%)$. The levels of resistance to ciprofloxacin and nalidixic acid are mostly moderate to very high within individual European countries, although some do not detect resistance or report low resistance to these compounds as we do in our study [19]. This considerable disparity in resistance to ciprofloxacin and nalidixic acid among Salmonella isolates, from different countries, may reflect the variability of serovars of Salmonella spp. included in the analyses of the different countries. Although antimicrobial use for animals is under veterinary prescription control in Greece, as in all European countries, farmers still sometimes use unprescribed antimicrobials for treatment or as growth promoters in cattle, poultry and swine. This practice leads into a possibility that bacterial resistance developing in the food animals' transfers to the human population thus posing a risk for public health by spreading of the resistance [28].

Cluster analysis did not reveal close genetic relationships between human and animal strains belonging to the same serovars. In most of the cases, distinct clusters were observed between human and non-human isolates, this can be clearly shown for $S$. Typhimurium, $S$. Blockley and $S$. Hadar. We suggest that this could be due to the isolates are epidemiologically unrelated.

Diversity among PFGE types varies for each particular serovar. It is well-known that $S$. Enteritidis is a clonal serovar. According to Pulsenet Europe database, which consists of 11,939 strains, 68 distinct and 509 unnamed profiles are present. Pulsenet PFGE-type SENTXB.0001 is predominant (56\%), the top 5 PFGE-types are representing $86 \%$ of all the isolates (data not shown). $S$. Hadar is also not a diverse serovar in Europe as in Greece; Pulsenet profile SHADXB.0001 is ranking first $(>50 \%$ of all isolates in Europe), the top five PFGE profiles represent 65\% of all isolates [11]. On the contrary, $S$. Typhimurium is a very diverse serovar, according to Pulsenet Europe database consisting of 6,027 strains divided into 275 distinct profiles and 1,554 unnamed profiles. The most prevalent PFGE profile is STMXB.0061 representing 679 out of 6,027 isolates (11\%) and the top five PFGE types are representing 1,887 isolates (31\%) (Data not shown)

This study indicates that municipal wastewater would be of interest to further monitor the community prevalence of subclinical or non-reported $S$. Enterica infections. Wastewater samples may be good indicators of emerging antimicrobial resistance in the community as was shown by the detection of glycopeptide resistance in Enterococcus faecium in Germany [29]. The use of wastewater sampling could be incorporated into national surveillance programs to monitor $S$. Enterica, as well as other pathogens, to obtain further knowledge of serovar shifts and their resistance patterns.

\section{Authors' Contributions}

TP has designed and performed all experiments under supervision of $\mathrm{AZ}$ and $\mathrm{AV}$. AZ, AV and GM provided the strains. GM, GV and VG have contributed in sample preparation and experimental procedure. EP participated in the manuscript preparation and advice during the work. All authors participated in draft and revision of the manuscript. All authors read and approved the final manuscript.

\section{Acknowledgments}

The authors gratefully acknowledge the personnel of National Veterinary Reference Centre for Salmonella, in Chalkis-Greece and also the personnel of National Reference Centre for SalmonellaCentral Public Health Laboratory, in Vari-Greece for serotyping all the veterinary and the human isolates, 
respectively. We also thank the administration and personnel of the Veterinary Research Institute (VRINAGREF) - Thessaloniki for their valuable cooperation and also for laboratory assistance in PFGE typing. This work was funded from Veterinary Research Institute of Thessaloniki (VRI-NAGREF).

\section{Competing Interests} interests.

The authors declare that they have no competing

\section{References}

1. European Food Safety Authority and European Centre for Disease Prevention and Control. The European Union summary report on trends and sources of zoonoses, zoonotic agents and food-borne outbreaks in 2013. EFSA J 2015;13:3991.

2. Jackson BR, Griffin PM, Cole D, Walsh KA, Chai SJ. Outbreak-associated Salmonella Enterica serotypes and food Commodities, United States, 1998-2008. Emerg Infect Dis 2013;19:1239-44.

3. Howard I, Espigares E, Lardelli P, Martín JL, Espigares M. Evaluation of microbiological and physicochemical indicators for wastewater treatment. Environ Toxicol 2004;19:241-9.

4. Oosterom J. Epidemiological studies and proposed preventive measures in the fight against human Salmonellosis. Int J Food Microbiol 1991;12:41-51.

5. Beuchat LR, Ryu JH. Produce handling and processing practices. Emerg Infect Dis 1997;3:459-65.

6. Threlfall EJ. Antimicrobial drug resistance in Salmonella: problems and perspectives in food- and water-borne infections. FEMS Microbiol Rev 2002;26:141-8.

7. Hohmann EL. Nontyphoidal salmonellosis. Clin Infect Dis 2001;32:263-9.

8. Threlfall EJ, Ward LR, Skinner JA, Graham A. Antimicrobial drug resistance in non-typhoidal salmonellas from humans in England and Wales in 1999: Decrease in multiple resistance in Salmonella Enterica serotypes Typhimurium, Virchow, and Hadar. Microb Drug Resist 2000;6:319-25.

9. Parry CM. Antimicrobial drug resistance in Salmonella Enterica. Curr Opin Infect Dis 2003;16:467-72.

10. Hopkins KL, Peters TM, de Pinna E, Wain J. Standardisation of multilocus variable-number tandem-repeat analysis (MLVA) for subtyping of Salmonella Enterica serovar Enteritidis. Euro Surveill 2011;16. pii:19942.

11. Papadopoulos T, Petridou E, Zdragas A, Nair S, Peters T, de Pinna E, et al. Phenotypic and molecular characterisation of multidrug-resistant Salmonella Enterica serovar Hadar in Greece, from 2007 to 2010. Clin Microbiol Infect 2015;21:149e1-4.

12. Foley SL, Zhao S, Walker RD. Comparison of molecular typing methods for the differentiation of Salmonella foodborne pathogens. Foodborne Pathog Dis 2007;4:253-76.

13. Clinical and Laboratory Standards Institute. Performance Standards for Antimicrobial Disk and Dilution Susceptibility Tests for Bacteria Isolated from Animals Approved Standard CLSI Document VET01-A4. In Approved Standard. $4^{\text {th }}$ ed. Wayne, PA, USA: CLSI; 2013.

14. The European Committee on Antimicrobial Susceptibility Testing-EUCAST. In: The European Committee on Antimicrobial Susceptibility Testing - EUCAST. European Society of Clinical Microbiology and Infectious Diseases; 2014.

15. Magiorakos AP, Srinivasan A, Carey RB, Carmeli Y, Falagas ME, Giske CG, et al. Multidrug-resistant, extensively drug-resistant and pandrug-resistant bacteria: An international expert proposal for interim standard definitions for acquired resistance. Clin Microbiol Infect 2012;18:268-81.

16. Hunter SB, Vauterin P, Lambert-Fair MA, Van Duyne MS, Kubota K, Graves L, et al. Establishment of a universal size standard strain for use with the pulsenet standardized pulsed-field gel electrophoresis protocols: Converting the national databases to the new size standard. J Clin Microbiol 2005;43:1045-50.

17. Voetsch AC1, Van Gilder TJ, Angulo FJ, Farley MM, Shallow S, Marcus R, et al. Food net estimate of the burden of illness caused by nontyphoidal salmonella infections in the United States. Clin Infect Dis 2004;38:S127-34.

18. Mellou K, Sideroglou T, Kallimani A, Potamiti-Komi M, Pervanidou D, Lillakou E, et al. Evaluation of underreporting of Salmonellosis and Shigellosis hospitalised cases in Greece, 2011: Results of a capture-recapture study and a hospital registry review. BMC Public Health 2013;13:875.

19. European Food Safety Authority and European Centre for Disease Prevention and Control. The European Union summary report on antimicrobial resistance in zoonotic and indicator bacteria from humans, animals and food in 2012. EFSA J 2014;12:3590.

20. European Food Safety Authority and European Centre for Disease Prevention and Control. The European Union Summary report on trends and sources of zoonoses, zoonotic agents and food-borne outbreaks in 2012. EFSA J 2014; $12: 312$.

21. National School of Public Health and Hellenic Center for Disease Control and Prevention. The Greek System for the Surveillance of Antimicrobial Resistance. National School of Public Health and Hellenic Center for Disease Control and Prevention; 2015.

22. Espigares E, Bueno A, Espigares M, Gálvez R. Isolation of Salmonella serotypes in wastewater and effluent: Effect of treatment and potential risk. Int J Hyg Environ Health 2006;209:103-7.

23. Berge AC, Dueger EL, Sischo WM. Comparison of Salmonella Enterica serovar distribution and antibiotic resistance patterns in wastewater at municipal water treatment plants in two California cities. J Appl Microbiol 2006;101:1309-16.

24. Catalao Dionisio LP, Joao M, Ferreiro VS, Fidalgo ML, García Rosado ME, Borrego JJ. Occurrence of Salmonella spp in estuarine and coastal waters of Portugal. Antonie Van Leeuwenhoek 2000;78:99-106.

25. Polo F, Figueras MJ, Inza I, Sala J, Fleisher JM, Guarro J. Prevalence of Salmonella serotypes in environmental waters and their relationships with indicator organisms. Antonie Van Leeuwenhoek 1999;75:285-92.

26. Iossifidou EG, Abrahim A, Soultos ND, Triantafillou EA, Koidis PA. Antimicrobial resistance profiles in Salmonella spp. Escherichia coli and isolates from Turkey samples in Northern Greece. Ann Microbiol 2012;62:623-8.

27. Zdragas A, Mazaraki K, Vafeas G, Giantzi V, Papadopoulos T, Ekateriniadou L. Prevalence, seasonal occurrence and antimicrobial resistance of Salmonella in poultry retail products in Greece. Lett Appl Microbiol 2012;55:308-13.

28. Ungemach FR, Müller-Bahrdt D, Abraham G. Guidelines for prudent use of antimicrobials and their implications on antibiotic usage in veterinary medicine. Int J Med Microbiol 2006;296 Suppl 41:33-8.

29. Klare I, Heier H, Claus H, Witte W. Environmental strains of Enterococcus faecium with inducible high-level resistance to glycopeptides. FEMS Microbiol Lett 1993;106:23-9. 